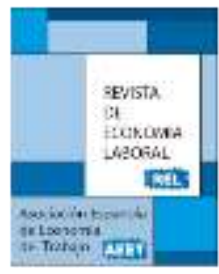

\title{
OFERTA Y DEMANDA DE EMPLEO PARA LA PRODUCCIÓN INTERNACIONAL DE BIENES Y SERVICIOS FINALES: MÉXICO Y CHINA, 1996-2011 1
}

\author{
Manuel García-Ramos ${ }^{2}$ \\ Instituto de Investigaciones Económicas (UNAM).
}

Recibido Septiembre 2019; Aceptado Noviembre 2019

\section{Resumen}

Con base en los datos de Comercio en Empleo (TiM), los objetivos de este documento son: mapear la oferta/demanda de empleo para la producción internacional de bienes y servicios finales y, de esta forma, cuantificar la importancia relativa de México y China. Los resultados sugieren que el empleo de China y México es dependiente de la demanda internacional de bienes y servicios finales, pero independiente del empleo externo para satisfacer su demanda final; sin embargo, comparado con otros países, el resto del mundo requiere más empleo chino para satisfacer su demanda final total; además, la proporción de personas empleadas en la producción de bienes y servicios finales para Estados Unidos en China fue la más alta.

Palabras clave: empleo, insumo-producto, economía de la complejidad, comercio internacional, división internacional del trabajo.

Clasificación JEL: C67; E24; F14; F16; R15

\section{Abstract}

Based on the Trade in eMployment (TiM) Database, the objectives of this document are: to map the supply/demand of employment for the international production of final goods and services and quantify the relative importance of Mexico and China. The results suggest that the employment of China and Mexico is dependent on international demand for final goods and services, but independent of foreign employment to satisfy their final demand. However, compared to other countries, the rest of the world requires more chinese employment to satisfy its total final demand; also, the proportion of employed people in the production of final goods and services for the United States in China was the highest.

Key words: employment, input-output, economics of complexity, international trade, international division of labour.

JEL Classification: C67; E24; F14; F16; R15

1 Este trabajo se llevó a cabo gracias al "Programa de Becas para investigadores sobre China", Centro de Estudios China-México (UNAM).

${ }^{2}$ Correo de contacto: sauceverde@hotmail.com 


\section{Introducción}

Existe una estrecha relación entre el comercio internacional y el empleo (UNCTAD, 2013; Jansen et al, 2011). Como señalan Schwarzer (2016), Jiang (2015) y McMillan y Verduzco (2011), ceteris paribus, la exportación de bienes y servicios finales genera empleo interno, mientras que la importación, empleo extranjero. Sin embargo, debido a la fragmentación internacional de la producción, algunos países exportan insumos para que el resto del mundo produzca sus exportaciones, mientras que otros importan insumos para producir sus exportaciones (García-Ramos, 2018; Gereffi y Fernandez-Stark, 2011). Es decir, tanto la exportación como la importación de bienes y servicios finales generan simultáneamente empleo interno y extranjero.

Lo anterior implica que, por un lado, los usos que un país puede darle a su empleo interno total son satisfacer la demanda final interna y/o la demanda final externa y que, por otro lado, el origen del empleo total necesario para satisfacer su propia demanda final son empleo interno y/o empleo externo. Por tanto, resulta necesario analizar la oferta y la demanda de empleo para la producción internacional de bienes y servicios finales como un sistema simultáneo, heterogéneo y entretejido; es decir, complejo.

Bajo un enfoque Neoestructuralista (Bielschowsky, 2013; Sunkel y Paz, 1970; Prebisch, 1949), asumimos que en el comercio internacional los países periféricos (con estructuras económicas especializadas y heterogéneas) y los países centrales (con estructuras económicas diversificadas y homogéneas) enfrentan problemas cualitativa y cuantitativamente distintos; sin embargo, no pueden estudiarse de forma separada, ya que las interrelaciones entre ellos determinan el comportamiento dinámico de la fragmentación internacional de la producción. Al igual que Dussel y Armony (2018), este documento enfatiza que la relación entre comercio internacional y empleo es un tema poco analizado (en especial, la importancia relativa de México y China); no obstante, su creciente y dinámica relevancia en términos cuantitativos y cualitativos.

Con base en los datos de Comercio en Empleo (TiM) de la OECD/WTO (2018) de 1995 a 2011, los objetivos de esta investigación son: mapear la oferta/demanda de empleo para la producción internacional de bienes y servicios finales y, de esta forma, cuantificar la importancia relativa de México y China. Este documento está estructurado de la siguiente manera: la sección 2 explica la metodología 
seguida para llevar a cabo los objetivos; la sección 3 presenta los resultados; y la sección final concluye.

\section{Métodos}

Es posible al analizar la oferta y demanda de empleo para la producción internacional de bienes y servicios finales como un sistema insumo-producto (Gibson, 2011) y, posteriormente, como una red compleja a través de un grafo ponderado dirigido (Barrat et al, 2004). El estudio de las redes complejas pertenece a la Teoría de Gafos y ésta, a su vez, al Análisis de Sistemas Complejos (Albert y Barabási, 2002). La representación gráfica de la oferta/demanda de empleo para la producción internacional de bienes y servicios finales, basada en redes complejas, es una herramienta poderosa para visualizar y analizar las características topológicas de este mercado: a través de su mapeo se pueden revelar patrones sistemáticos latentes que "emergen" en los datos (Maurer y Degain; 2010; Christensen y Albert, 2007). La metodología propuesta tiene como base los trabajos de García-Ramos y Fujii-Gambero (2017), Taglioni y Winker (2016), Cerina et al. (2015).

\subsection{Oferta y demanda de empleo para la producción internacional de bienes y servicios finales}

En la Tabla 1 se muestran la oferta (uso) y la demanda (origen) de empleo para la producción internacional de bienes y servicios finales. El elemento $e_{i j}$ es el número de personas en el país $i$ empleadas en la producción de bienes y servicios finales para el país $j$.

Por filas, los usos que el país $i$ puede darle a su empleo total $\left(y_{i}\right)$ son satisfacer la demanda final interna $\left(n_{i}\right)$ y/o la demanda final externa $\left(x_{i}\right)$; de esta forma:

$$
y_{i}=n_{i}+x_{i}
$$

Donde $x_{i}=\sum_{j=1}^{n} e_{i j}$

Por columnas, el origen del empleo total $\left(y_{j}\right)$ necesario para satisfacer la demanda final total del país $\mathrm{j}$ es empleo interno $\left(d_{j}\right)$ y/o empleo externo $\left(m_{j}\right)$; de esta forma: 


$$
y_{j}=d_{j}+m_{j}
$$

Donde $m_{j}=\sum_{i=1}^{n} e_{i j}$

En términos generales, la Tabla 1 muestra que la producción internacional de bienes y servicios finales tiene como base la división internacional del trabajo, la cual opera en equilibrio global; es decir, la oferta es igual a la demanda: $\sum_{i=1}^{n} x_{i}+\sum_{i=1}^{n} n_{i}=\sum_{j=1}^{n} m_{j}+\sum_{j=1}^{n} d_{j}$. Además, se cumple que $\sum_{i=1}^{n} x_{i}=\sum_{j=1}^{n} m_{j} \sum_{i=1}^{n} n_{i}=\sum_{j=1}^{n} d_{j} \quad \sum_{i=1}^{n} y_{i}=\sum_{j=1}^{n} y_{j}$, aunque esto no se verifique para cada país; en otras palabras, existen desequilibrios múltiples, ya que $x_{i} \neq m_{j}$ y $n_{i} \neq d_{j}$, cuando $\mathrm{i}=\mathrm{j}$. Lo anterior es afín al enfoque Neoestructuralista: los mercados internacionales son heterogéneos y no integrados; por tanto, se reconocen los desequilibrios estructurales.

Las expresiones (1) y (2) se pueden generalizar como dos sistemas de ecuaciones simultáneas lineales, en los cuales cada ecuación muestra la oferta (3) y la demanda (4) de empleo para la producción internacional de bienes y servicios finales, respectivamente:

$$
\begin{gathered}
\mathbf{y}^{T}=\mathbf{i}^{T} \mathbf{E}+\mathbf{d}^{T} \\
\mathbf{y}=\mathbf{E} \mathbf{i}+\mathbf{n}
\end{gathered}
$$

Donde

$$
\mathbf{E}=\left\{e_{i j} \mid e_{i j}=0, \forall i=j ; \text { de otra forma } \mathrm{e}_{i j}>0\right\} \text {, }
$$

$$
\mathbf{d}^{T}=\left[\begin{array}{lll}
d_{1} & \cdots & d_{n}
\end{array}\right], \mathbf{n}^{T}=\left[\begin{array}{lll}
n_{1} & \cdots & n_{n}
\end{array}\right], \mathbf{y}^{T}=\left[\begin{array}{lll}
y_{1} & \cdots & y_{n}
\end{array}\right], \mathbf{i}^{T}=\left[\begin{array}{lll}
1 & \cdots & 1
\end{array}\right] \mathrm{y}
$$

T indica transposición.

Si consideramos que los elementos de la matriz E son proporciones fijas y constantes que describen la oferta y la demanda de empleo para la producción internacional de bienes y servicios finales, respectivamente, entonces podemos definir:

$$
\alpha_{i j}=e_{i j} / y_{i}
$$




$$
\beta_{i j}=e_{i j} / y_{j}
$$

Tabla 1. Oferta y demanda de empleo para la producción internacional de bienes y servicios finales

\begin{tabular}{|c|c|c|c|c|c|c|c|c|c|c|}
\hline & \multicolumn{6}{|c|}{ País de destino } & \multicolumn{3}{|c|}{ Oferta de empleo } \\
\hline & & $p_{1}$ & $p_{2}$ & $\cdots$ & $p_{j}$ & $\cdots$ & $p_{n}$ & $\begin{array}{c}\text { Para demanda } \\
\text { externa }\end{array}$ & $\begin{array}{c}\text { Para demanda } \\
\text { interna }\end{array}$ & Total \\
\hline \multirow{6}{*}{ 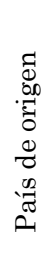 } & $p_{1}$ & 0 & $e_{12}$ & $\cdots$ & $e_{1 j}$ & $\cdots$ & $e_{1 n}$ & $x_{1}$ & $n_{1}$ & $y_{1}$ \\
\hline & $p_{2}$ & $e_{21}$ & $O$ & $\cdots$ & $e_{2 j}$ & $\cdots$ & $e_{2 n}$ & $x_{2}$ & $n_{2}$ & $y_{2}$ \\
\hline & $\vdots$ & $\vdots$ & $\vdots$ & $\ddots$ & $\vdots$ & & $\vdots$ & $\vdots$ & $\vdots$ & $\vdots$ \\
\hline & $p_{i}$ & $e_{i 1}$ & $e_{i 2}$ & $\cdots$ & 0 & $\cdots$ & $e_{i n}$ & $x_{i}$ & $n_{i}$ & $y_{i}$ \\
\hline & $\vdots$ & $\vdots$ & $\vdots$ & & & $\ddots$ & $\vdots$ & : & $\vdots$ & $\vdots$ \\
\hline & $p_{n}$ & $e_{n 1}$ & $e_{n 2}$ & $\cdots$ & $e_{n j}$ & $\cdots$ & 0 & $x_{n}$ & $n_{n}$ & $y_{n}$ \\
\hline \multirow{3}{*}{ 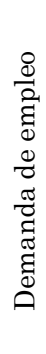 } & Externo & $m_{1}$ & $m_{2}$ & $\cdots$ & $m_{j}$ & $\cdots$ & $m_{n}$ & $\sum_{i=1} x_{i}=\sum_{i=1} m_{j}$ & & \\
\hline & Interno & $d_{1}$ & $d_{2}$ & $\cdots$ & $d_{j}$ & $\cdots$ & $d_{n}$ & & $\sum_{i=1}^{n} n_{i}=\sum_{i=1}^{n} d$ & \\
\hline & Total & $y_{1}$ & $y_{2}$ & $\cdots$ & $y_{j}$ & $\cdots$ & $y_{n}$ & & & $=$ \\
\hline
\end{tabular}

La expresión (5) muestra el número de personas en el país i empleadas en la producción internacional de bienes y servicios finales para el país j como proporción del empleo total del país i (composición de la oferta), mientras que la expresión (6) muestra el número de personas en el país i empleadas en la producción internacional de bienes y servicios finales para el país $\mathrm{j}$ como proporción del empleo total del país $\mathrm{j}$ (composición de la demanda). A través de (5) y (6) podemos reescribir (3) y (4) como:

$$
\begin{aligned}
\mathbf{y}^{T}(\mathbf{I}-\mathbf{A}) & =\mathbf{d}^{T} \\
(\mathbf{I}-\mathbf{B}) \mathbf{y} & =\mathbf{n}
\end{aligned}
$$




$$
\begin{aligned}
& \text { Las matrices } \mathbf{A}=\left\{\alpha_{i j} \mid \alpha_{i j}=0, \forall i=j \text {; de otra forma } \alpha_{i j} \geq 0\right\} \quad \mathrm{y} \\
& \mathbf{B}=\left\{\beta_{i j} \mid \beta_{i j}=0, \forall i=j \text {; de otra forma } \beta_{i j} \geq 0\right\} \\
& \text { desperiben, } \\
& \text { para producir una unidad adicional de bienes y servicios finales. Es } \\
& \text { importante destacar que } a_{i j} \times\left(y_{i} / Y\right)=b_{i j} \times\left(y_{j} / Y\right)=e_{i j} / Y \text {; por tanto, } \\
& \text { podemos definir } \mathbf{W}=\left\{w_{i j} \mid w_{i j}=e_{i j} / Y\right\} .
\end{aligned}
$$

\subsection{Los mapas de la oferta/demanda de empleo para la producción internacional de bienes y servicios finales}

Los grafos son estructuras geométricas de datos no lineales que permiten visualizar y analizar las interrelaciones complejas entre los elementos heterogéneos de un conjunto (red). El grafo de una red se constituye por nodos, aristas y pesos. El siguiente grafo ponderado dirigido representa la red compleja del comercio de empleo para la producción internacional de bienes y servicios finales:

$$
G(N, R, W)
$$

Donde $N=\left\{p_{1}, \ldots, p_{n}\right\}$ es el conjunto de $\mathrm{n}$ países o nodos que participan en la oferta/demanda de empleo para la producción internacional de bienes $y$ servicios finales; $R=\left\{r_{i \rightarrow j} \in N^{2} \mid r_{i \rightarrow j} \in \mathbf{\Xi}(G)=\left\{\begin{array}{l}1, \text { si } w_{i j} \in \mathbf{W} \geq 0.01 \\ 0, \text { de otra forma }\end{array}\right\}\right.$

aristas 0 relaciones ordenadas entre pares de nodos; $W=\left\{w_{i \rightarrow j} \in R \mid w_{i \rightarrow j}=w_{i j} \in \mathbf{W}\right\}$ es el conjunto de pesos asociados a las aristas; y $\Xi(\mathrm{G})$ es la matriz de adyacencia de $G(N, R, W)$.

Para examinar con mayor profundidad el grafo (9), los siguientes indicadores clasifican a los países a través de su posición en la oferta/demanda de empleo para la producción internacional de bienes y servicios finales: 
1. Demanda de empleo, $\left(\sum_{i} \beta_{i j}\right) \times\left(y_{j} / Y\right)$, para todo $\beta_{i j}>0.01$. Este indicador mide la proporción de personas empleadas en el resto del mundo en la producción internacional de bienes y servicios finales para el país i, respecto al empleo total mundial. Si $D_{i} \leq$ promedio, el país i demanda poco empleo externo; y, si $D_{i}>$ promedio, el país i demanda mucho empleo externo.

2. Oferta de empleo, $O_{i}=\left(\sum_{j} \alpha_{i j}\right) \times\left(y_{i} / Y\right)$, para todo $\alpha_{i j}>0.01$. Este indicador mide la proporción de personas empleadas en el país i en la producción internacional de bienes y servicios finales para el resto del mundo, respecto al empleo total mundial. Si $O_{i} \leq$ promedio, el resto del mundo demanda poco empleo del país i; y, si $O_{i}>$ promedio, el resto del mundo demanda mucho empleo del país i.

3. Posición, $P_{i}=O_{i}-D_{i}$. Este indicador mide la posición del país i en la oferta /demanda de empleo para la producción internacional de bienes y servicios finales. Si $P_{i}<0$, el país i es independiente de la demanda final externa, pero dependiente del empleo externo para satisfacer su propia demanda final; si $P_{i}>0$, el país i es dependiente de la demanda final externa, pero independiente del empleo externo para satisfacer su propia demanda final. Los países con $P_{i}>0$ son tipo I, mientras que los países con $P_{i}<0$ son tipo II.

\section{Resultados}

La falta de datos recientes nos obliga limitar el alcance del análisis empírico, un hecho que puede ser un obstáculo importante en la búsqueda de tendencias y relaciones sistemáticas que puedan ser interpretadas plenamente en un marco teórico-económico; además, la base de datos TiM (Trade in eMployment) utiliza los resultados de la Inter-Country Input-Output (ICIO) 2015.

\subsection{Indicadores básicos, 1996-2011}


La Tabla 2 muestra los indicadores básicos de los países con $y_{i} / Y>1 \%$. En promedio, el peso de China (CHN), India (IND), Estados Unidos (USA), Indonesia (IDN), Brasil (BRA), Japón (JPN), Rusia (RUS), Alemania (DEU), México (MEX), Reino Unido (GBR), Francia (FRA), Italia (ITA), Turquía (TUR), España (ESP) y Corea (KOR) en el empleo total mundial fue aproximadamente el 90\%. Mientras que el peso del empleo de China fue, en promedio, 36.74\%, el de México fue del $2 \%$. Cabe destacar que el único país con un peso semejante al de China fue India $(21.52 \%)$.

El resto de los indicadores señalan que, en 1996, el $0.30 \%$ del empleo necesario para satisfacer la producción de bienes y servicios finales de China fue de origen externo y $99.7 \%$ de origen interno, mientras que México necesitó $4.34 \%$ de empleo externo y $95.66 \%$ de empleo interno. Por otro lado, el $7.39 \%$ del empleo de China fue para satisfacer la demanda final externa y el $92.61 \%$ para la demanda final interna, mientras que $14.91 \%$ del empleo de México fue para satisfacer la demanda final externa y $85.09 \%$ para la demanda final interna.

En contraste, en 2011, el 2.26\% del empleo necesario para satisfacer la producción de bienes y servicios finales de China fue de origen externo y $97.74 \%$ de origen interno, mientras que México necesitó $9.36 \%$ de empleo externo y $90.64 \%$ de empleo interno. Por otro lado, el $11.38 \%$ del empleo de China fue para satisfacer la demanda final externa y el $88.62 \%$ para la demanda final interna, mientras que $14.10 \%$ del empleo de México fue para satisfacer la demanda final externa y $85.90 \%$ para la demanda final interna. Estos resultados son congruentes con los hallazgos de Gallagher y Porzecanski (2008).

Es importante destacar que, de forma sistemática, el $44.53 \%$ del empleo necesario para satisfacer la producción de bienes y servicios finales de Estados Unidos fue externo y el 55.47\%, interno. Por otro lado, para satisfacer su demanda final India, Indonesia y Brasil necesitaron menos del 4\% de empleo externo; el resto de los países requiere más del $20 \%$. Además, el 25.30\% del empleo en Alemania fue para la demanda final externa, mientras que el $74.70 \%$ fue la demanda final interna. En términos generales, sólo en Estados Unidos, India y Brasil el 10\% de su empleo fue para satisfacer la demanda final externa.

La Tabla 3 muestra la clasificación presentada en la sección 2.2. En primer lugar, comparado con el resto de los países, el indicador Di sugiere que, en 1996, México fue un país que demandó poco empleo externo, mientras China fue uno que demandó más que el promedio; $0.08 \%$ y $0.11 \%$, respectivamente. En 2011, comparados con el resto del 
mundo, tanto México como China demandaron mucho empleo externo: $0.21 \%$ y $0.81 \%$, respectivamente.

El indicador Oi muestra que, en 1996, el resto del mundo demandó más empleo de China que de México para la producción internacional de bienes y servicios finales: $0.27 \%$ y $2.78 \%$, respectivamente. En 2011, para satisfacer la demanda final, el resto del mundo utilizó menos empleo de México que de China: $0.31 \%$ y $4.09 \%$, respectivamente.

Finalmente, el indicador Pi sugiere que, de forma sistemática, el empleo de China y México fue dependiente de la demanda internacional de bienes y servicios finales, pero independiente del empleo externo para satisfacer su propia demanda final; lo mismo sucedió para, India, Indonesia, Rusia y Brasil. En contraste, el empleo del resto de los países es independiente de la demanda internacional de bienes y servicios finales, pero dependiente del empleo externo para satisfacer su propia demanda final.

\subsection{México y China: los mapas de oferta/demanda de empleo para la producción internacional de bienes y servicios finales}

Las Figuras 1-8 muestran los mapas de la oferta/demanda de empleo para la producción internacional de bienes y servicios finales; 1996, 2001, 2006 y 2011, respectivamente. Para su construcción se utilizó el algoritmo Force Atlas 2 (Jacomy et al., 2014): los nodos se repelen/atraen entre sí como imanes, mientras que las aristas atraen a los nodos como resortes; estas fuerzas crean un movimiento que produce una espacialización legible de los datos. 
Tabla 2. Indicadores básicos, porcentajes, 1996-2011

\begin{tabular}{|c|c|c|c|c|c|c|c|c|c|c|c|c|c|c|c|}
\hline 1996 & $/ Y$ & $\mathrm{i} / \mathrm{yi}_{\mathrm{i}}$ & $\mathrm{x}_{\mathrm{i}} / \mathrm{yi}_{\mathrm{i}}$ & 001 & $\mathrm{y}_{\mathrm{i}} / \mathrm{Y}$ & $\mathrm{i} / \mathrm{yi}_{\mathrm{i}}$ & $\mathrm{xi}_{\mathrm{i}} / \mathrm{yi}_{\mathrm{i}}$ & 2006 & i/Y & $\mathrm{m}_{\mathrm{i}} / \mathrm{y}_{\mathrm{i}}$ & $\mathrm{xi} / \mathrm{yi}$ & 2011 & $\mathrm{yi}_{\mathrm{i}} / \mathrm{Y}$ & $\mathrm{mi}_{\mathrm{i}} / \mathrm{yi}$ & $\mathrm{i} / \mathrm{yi}$ \\
\hline $\mathrm{CHN}$ & 37.58 & 0.30 & 739 & $\mathrm{CHN}$ & 7.24 & 079 & 786 & $\mathrm{HN}$ & 6.21 & 1.25 & 15.45 & $\mathrm{CHN}$ & & & \\
\hline IND & 20.92 & 0.30 & 6.69 & & & .48 & & ND & 21.99 & 1.01 & & & 21.86 & 55 & \\
\hline USA & 7.04 & 33.37 & 7.20 & USA & 7.12 & 45.77 & 6.53 & USA & 7.09 & 56.68 & 6.52 & USA & 6.68 & 42.30 & 7.91 \\
\hline IDN & 4.70 & 2.76 & 15.49 & IDN & & 2.07 & & IDN & & & & & & & \\
\hline BRA & & 2.89 & 5.84 & & & & & & & & & & & & \\
\hline JPN & 3.67 & 41.53 & 6.32 & $\mathrm{JPN}$ & & 38.41 & 7.45 & US & 3.32 & 8.61 & & RUS & 3.32 & 11.58 & 15.16 \\
\hline RUS & 3.30 & 6.05 & 11 & RUS & & 508 & & $\mathrm{~N}$ & & & & JPN & & & 9.65 \\
\hline DEL & & & & & & & & & & & & & & & \\
\hline MEX & & & & & & & & & & & & & & & \\
\hline GBR & 1.42 & 36.70 & 18.69 & GBR & 1.42 & 46.78 & 16.96 & $R$ & 1.41 & 67.80 & & GBR & 1.38 & 50.90 & 19.69 \\
\hline FRA & 1.30 & 34.99 & 15.94 & FRA & 1.33 & & 18.72 & FRA & & & & FRA & 1.27 & & 17.17 \\
\hline ITA & & 34.64 & 18.57 & & & & & & & & & & & & \\
\hline TUR & 1.17 & 8.06 & 11.19 & $U \pi$ & 1.10 & 31.83 & & KOR & 1.12 & 46.46 & & & 1.14 & & 22.30 \\
\hline KOR & 1.14 & 36.62 & 17.21 & TUR & 1.10 & 6.34 & 18.42 & ESP & 0.99 & 44.81 & 18.16 & TUR & 1.13 & 19.94 & 13.00 \\
\hline
\end{tabular}

Fuente: OECD/WTO; cálculos del autor 
Tabla 3. Clasificación de países, porcentajes, 1996-2011

\begin{tabular}{|c|c|c|c|c|c|c|c|c|c|c|c|c|c|c|c|}
\hline \multicolumn{4}{|c|}{1996} & \multicolumn{4}{|c|}{2001} & \multicolumn{4}{|c|}{2006} & \multicolumn{4}{|c|}{2011} \\
\hline Tipo I & $\mathrm{D}_{\mathrm{i}}$ & $\mathrm{O}_{\mathrm{i}}$ & $\mathrm{P}_{\mathrm{i}}$ & Tipo I & $\mathrm{D}_{\mathrm{i}}$ & $\mathrm{O}_{\mathrm{i}}$ & $\mathrm{P}_{\mathrm{i}}$ & Tipo I & $\mathrm{D}_{\mathrm{i}}$ & $\mathrm{O}_{\mathrm{i}}$ & $\mathrm{P}_{\mathrm{i}}$ & Tipo I & $\mathrm{D}_{\mathrm{i}}$ & $\mathrm{O}_{\mathrm{i}}$ & $\mathrm{P}_{\mathrm{i}}$ \\
\hline $\mathrm{CHN}$ & 0.11 & 2.78 & 2.66 & $\mathrm{CHN}$ & 0.27 & 2.93 & 2.66 & $\mathrm{CHN}$ & 0.45 & 5.59 & 5.14 & $\mathrm{CHN}$ & 0.81 & 4.09 & 3.27 \\
\hline IND & 0.06 & 1.40 & 1.34 & IND & 0.10 & 1.53 & 1.43 & IND & 0.22 & 2.15 & 1.93 & IND & 0.36 & 2.48 & 2.12 \\
\hline IDN & 0.13 & 0.73 & 0.60 & IDN & 0.10 & 0.98 & 0.88 & IDN & 0.13 & 0.77 & 0.64 & IDN & 0.20 & 0.74 & 0.54 \\
\hline MEX & 0.08 & 0.27 & 0.19 & RUS & 0.17 & 0.65 & 0.48 & BRA & 0.16 & 0.54 & 0.38 & RUS & 0.38 & 0.50 & 0.12 \\
\hline RUS & 0.20 & 0.38 & 0.18 & BRA & 0.10 & 0.41 & 0.31 & RUS & 0.29 & 0.54 & 0.26 & MEX & 0.21 & 0.31 & 0.10 \\
\hline BRA & 0.11 & 0.23 & 0.12 & TUR & 0.07 & 0.20 & 0.13 & MEX & 0.24 & 0.29 & 0.05 & BRA & 0.29 & 0.39 & 0.10 \\
\hline TUR & 0.09 & 0.13 & 0.04 & MEX & 0.17 & 0.27 & 0.10 & Tipo II & $\mathrm{D}_{\mathrm{i}}$ & $\mathrm{O}_{\mathrm{i}}$ & $\mathrm{P}_{\mathrm{i}}$ & Tipo II & $\mathrm{D}_{\mathrm{i}}$ & $\mathrm{O}_{\mathrm{i}}$ & $\mathrm{P}_{\mathrm{i}}$ \\
\hline Tipo II & $\mathrm{D}_{\mathrm{i}}$ & $\mathrm{O}_{\mathrm{i}}$ & $\mathrm{P}_{\mathrm{i}}$ & Tipo II & $\mathrm{D}_{\mathrm{i}}$ & $\mathrm{O}_{\mathrm{i}}$ & $\mathrm{P}_{\mathrm{i}}$ & ESP & 0.45 & 0.18 & -0.27 & TUR & 0.23 & 0.15 & -0.08 \\
\hline ITA & 0.42 & 0.22 & -0.19 & KOR & 0.35 & 0.24 & -0.11 & KOR & 0.52 & 0.23 & -0.29 & KOR & 0.41 & 0.25 & -0.15 \\
\hline KOR & 0.42 & 0.20 & -0.22 & ITA & 0.48 & 0.24 & -0.25 & ITA & 0.57 & 0.24 & -0.33 & ITA & 0.53 & 0.23 & -0.30 \\
\hline FRA & 0.45 & 0.21 & -0.25 & FRA & 0.52 & 0.25 & -0.27 & FRA & 0.64 & 0.23 & -0.42 & DEU & 0.87 & 0.49 & -0.37 \\
\hline GBR & 0.52 & 0.27 & -0.26 & GBR & 0.66 & 0.24 & -0.42 & DEU & 0.99 & 0.47 & -0.52 & FRA & 0.63 & 0.22 & -0.42 \\
\hline DEU & 0.97 & 0.35 & -0.62 & DEU & 0.93 & 0.44 & -0.49 & GBR & 0.95 & 0.23 & -0.72 & GBR & 0.70 & 0.27 & -0.43 \\
\hline JPN & 1.52 & 0.23 & -1.29 & JPN & 1.28 & 0.25 & -1.04 & JPN & 1.28 & 0.33 & -0.95 & JPN & 1.01 & 0.29 & -0.72 \\
\hline USA & 2.35 & 0.51 & -1.84 & USA & 3.26 & 0.47 & -2.79 & USA & 4.02 & 0.46 & -3.55 & USA & 2.83 & 0.53 & -2.30 \\
\hline Promedio & 0.20 & 0.20 & & Promedio & 0.22 & 0.22 & & Promedio & 0.29 & 0.29 & & Promedio & 0.26 & 0.26 & \\
\hline
\end{tabular}


La lectura de cada mapa es la siguiente: 1) el tamaño de cada nodo es proporcional a la magnitud del indicador $O_{i}$; es decir, a la proporción de personas empleadas en el país i en la producción internacional de bienes y servicios finales para el resto del mundo, respecto al empleo total mundial. Cuanto mayor sea $O_{i}$, mayor será el tamaño del nodo; 2) el color de cada nodo es proporcional a la magnitud del indicador $D_{i}$; es decir, a la proporción de personas empleadas en el resto del mundo en la producción internacional de bienes y servicios finales para el país $i$, respecto al empleo total mundial. Cuanto mayor sea $D_{i}$, más oscuro será el color del nodo; 3) el grosor y la dirección de las aristas son proporcionales al elemento $w_{i j} \in \mathbf{W}$; es decir, a la proporción de personas empleadas en el país i en la producción de bienes y servicios finales para el país j, respecto al empleo total mundial. Por tanto, representan simultáneamente la oferta/demanda de empleo entre dos países. Cuanto mayor sea $W_{i j}$, más gruesa será la arista entre pares de nodos.

Los patrones sistemáticos más relevantes en las Figuras 1-8 son los siguientes: 1) comparado con el resto del mundo, China fue el país con el indicador $O_{i}$ más alto; es decir, fue el país con la proporción más alta de personas empleadas en la producción internacional de bienes y servicios finales para el resto del mundo; en otras palabras, el resto del mundo demandó mucho empleo chino para satisfacer su demanda final total; 2) Estados Unidos fue el país con el indicador $D_{i}$ más alto; es decir, la proporción más alta de personas en el resto del mundo empleadas en la producción internacional de bienes y servicios finales fue para Estados Unidos; en otras palabras, este país demandó mucho empleo externo para satisfacer su demanda final total; 3) la proporción de personas empleadas en China en la producción de bienes y servicios finales para Estados Unidos, respecto al empleo total mundial, fue la más alta; en segundo lugar, la de China $\rightarrow$ Japón; y 4) comparado con China, la participación de México en la red fue menor: en promedio, la China fue $8.55 \%$, mientras que la de México, 3.66\%. De acuerdo con Lall y Weiss (2007), a pesar de que China y México poseen estructuras de exportación similares y están integrados en redes productivas análogas, los patrones de ambos países descubren un caso clásico de comercio de un país en desarrollo y uno industrializado. 
Figura 1. México: oferta/demanda de empleo para la producción internacional de bienes y servicios finales, 1996

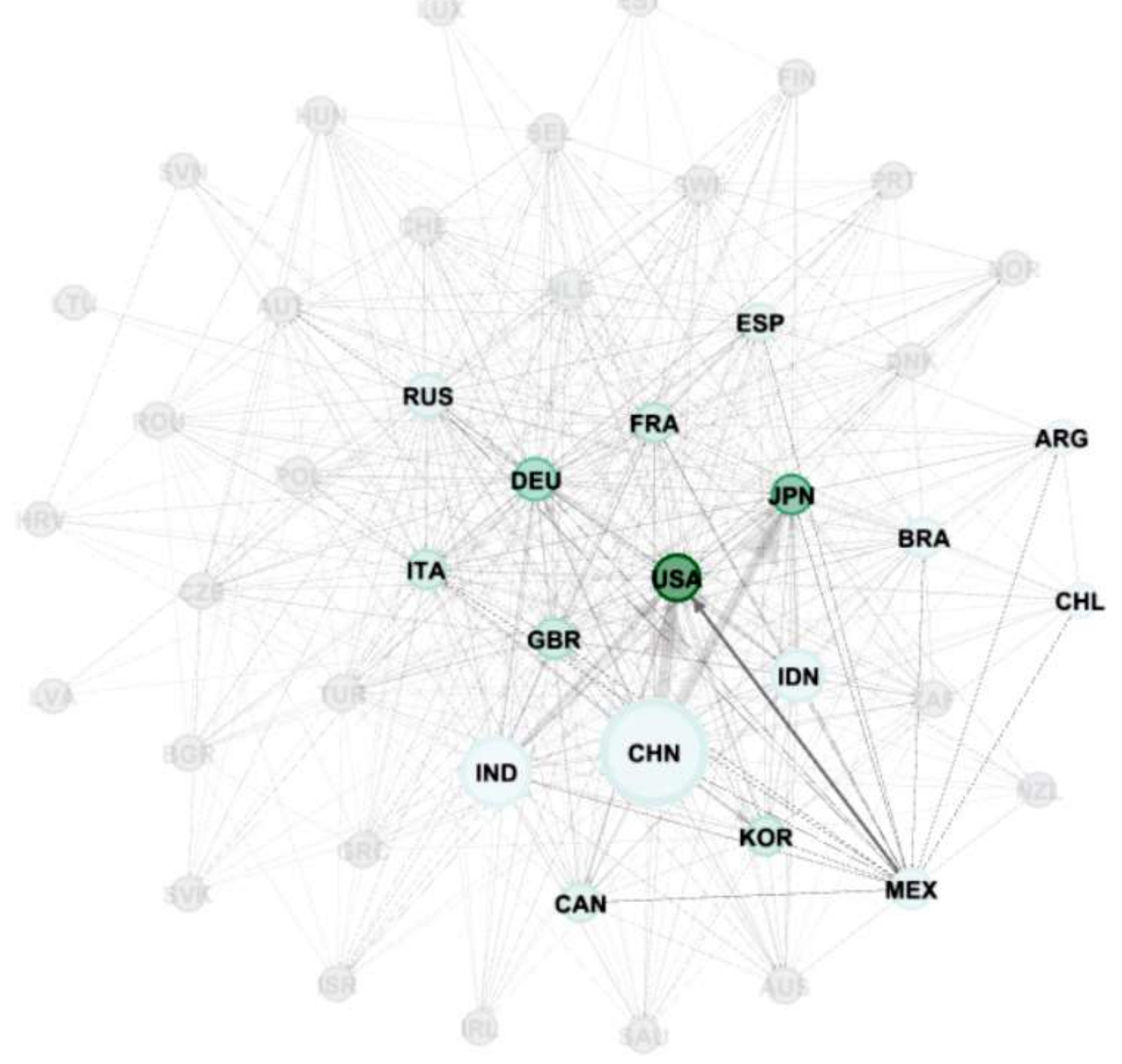

Fuente: OECD/WTO; cálculo del autor 
Figura 2. México: oferta/demanda de empleo para la producción internacional de bienes y servicios finales, 2001

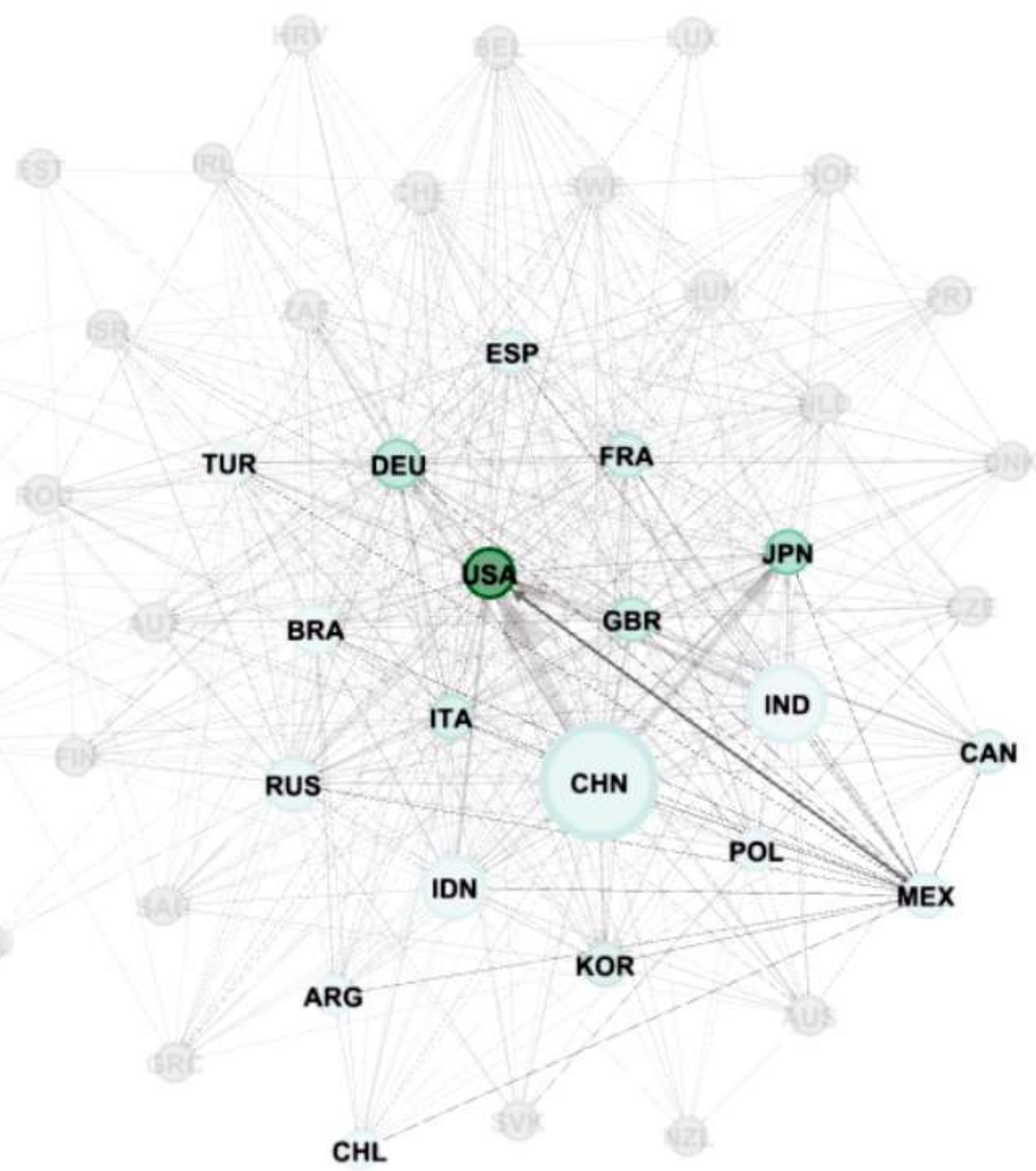

Fuente: OECD/WTO; cálculo del autor 
Figura 3. México: oferta/demanda de empleo para la producción internacional de bienes y servicios finales, 2006

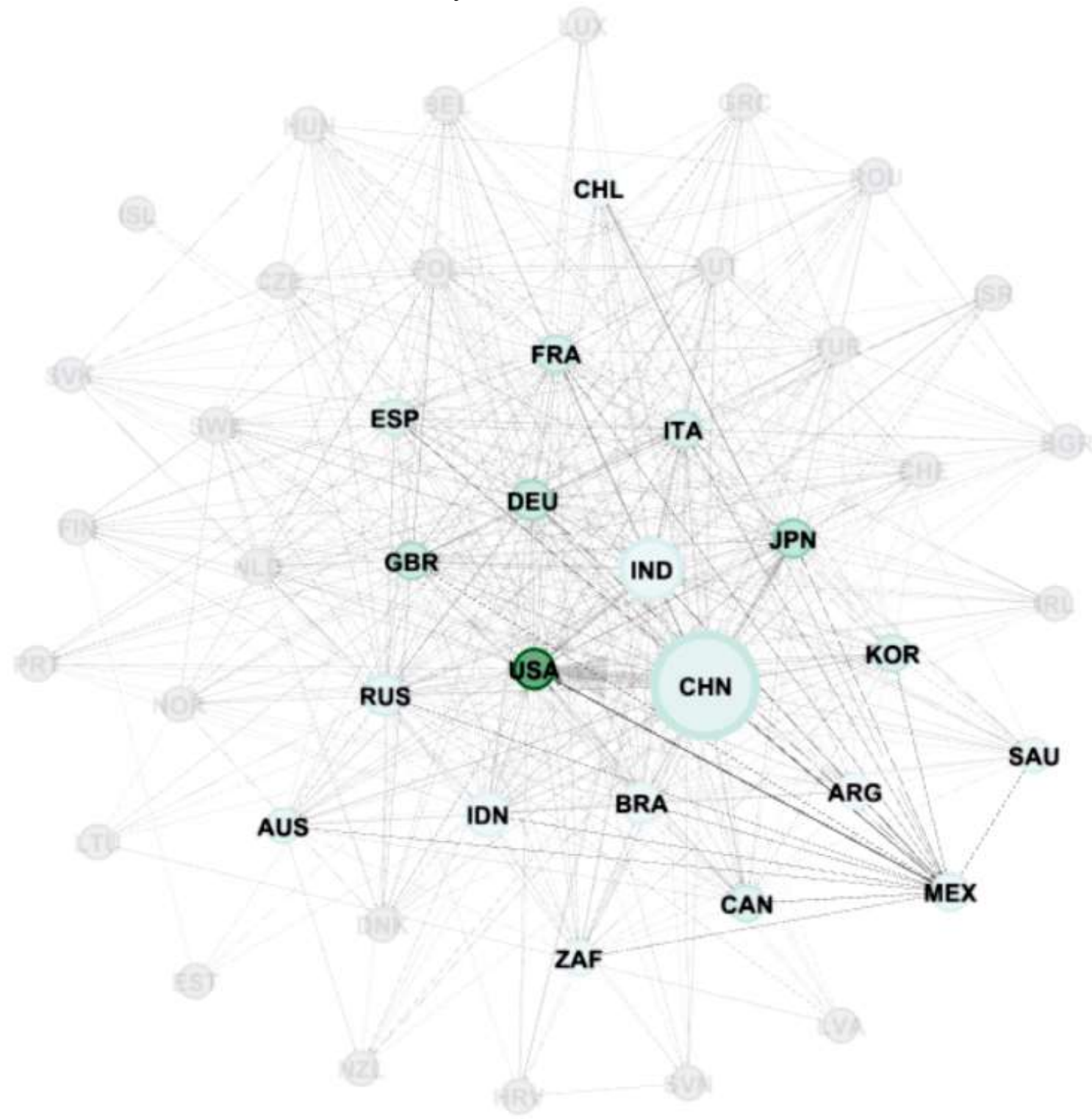

Fuente: OECD/WTO; cálculo del autor 
Figura 4. México: oferta/demanda de empleo para la producción internacional de bienes y servicios finales, 2011

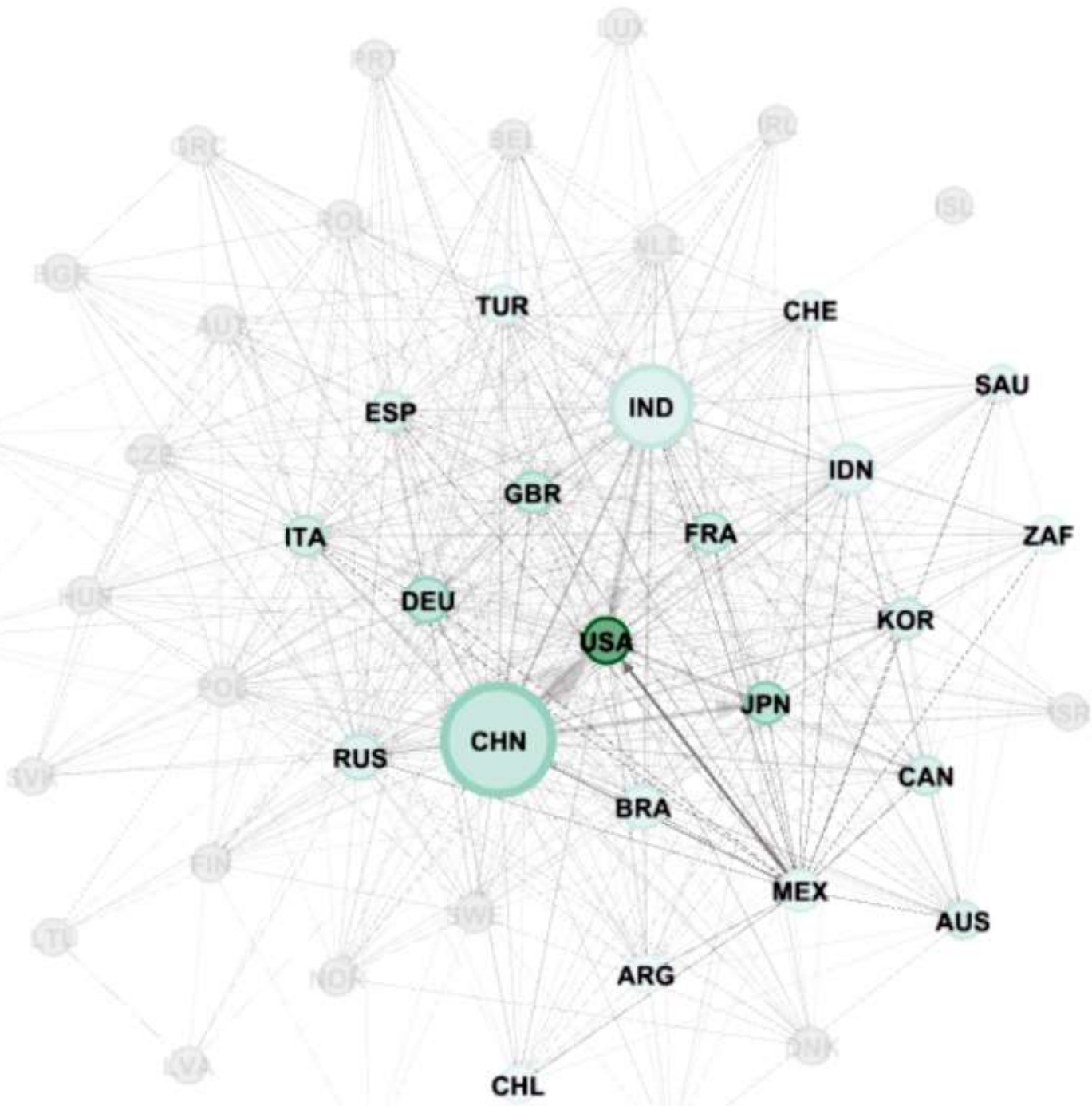

Fuente: OECD/WTO; cálculo del autor 
Figura 5. China: oferta/demanda de empleo para la producción internacional de bienes y servicios finales, 1996

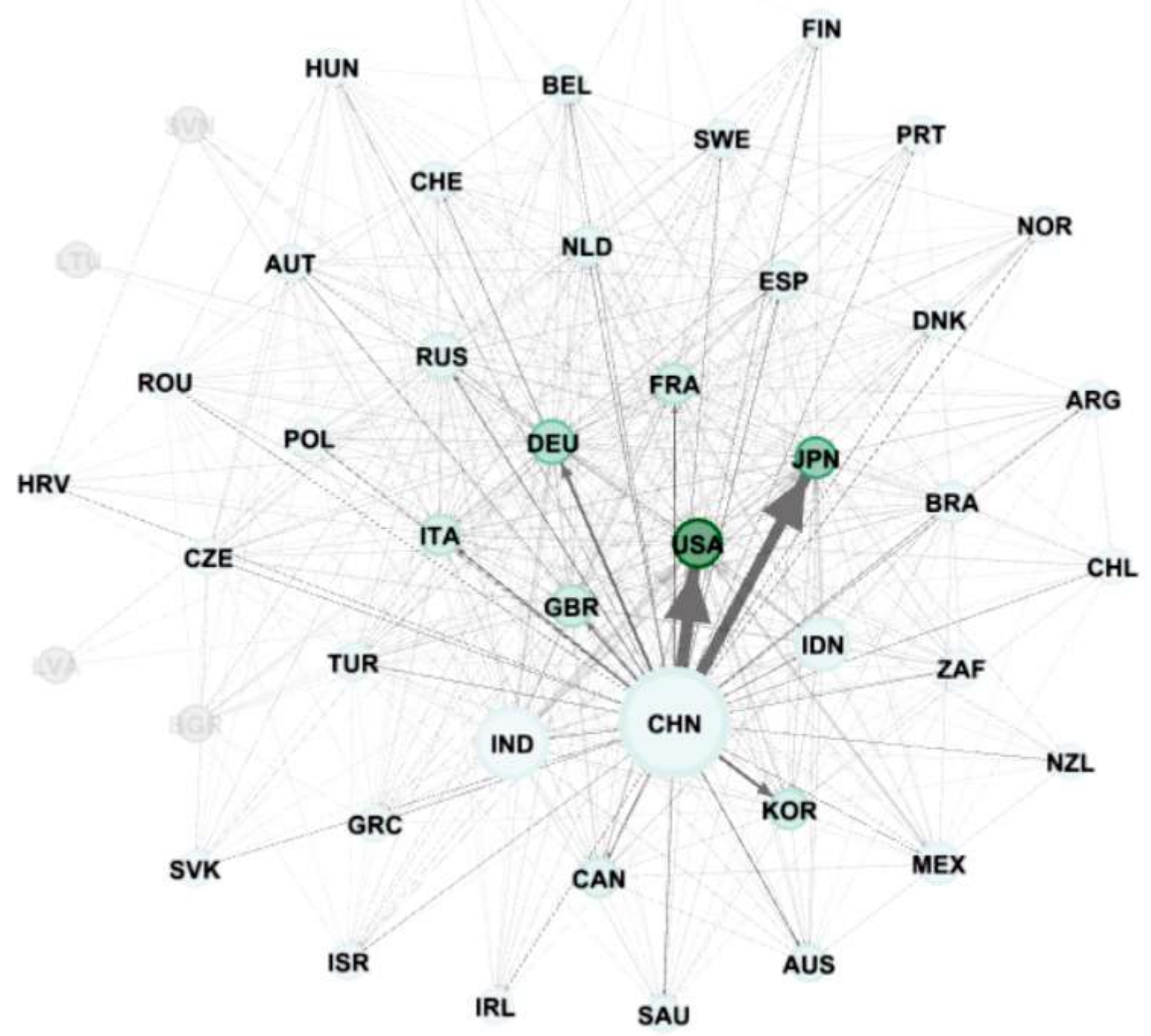

Fuente: OECD/WTO; cálculo del autor 
Figura 6. China: oferta/demanda de empleo para la producción internacional de bienes y servicios finales, 2001

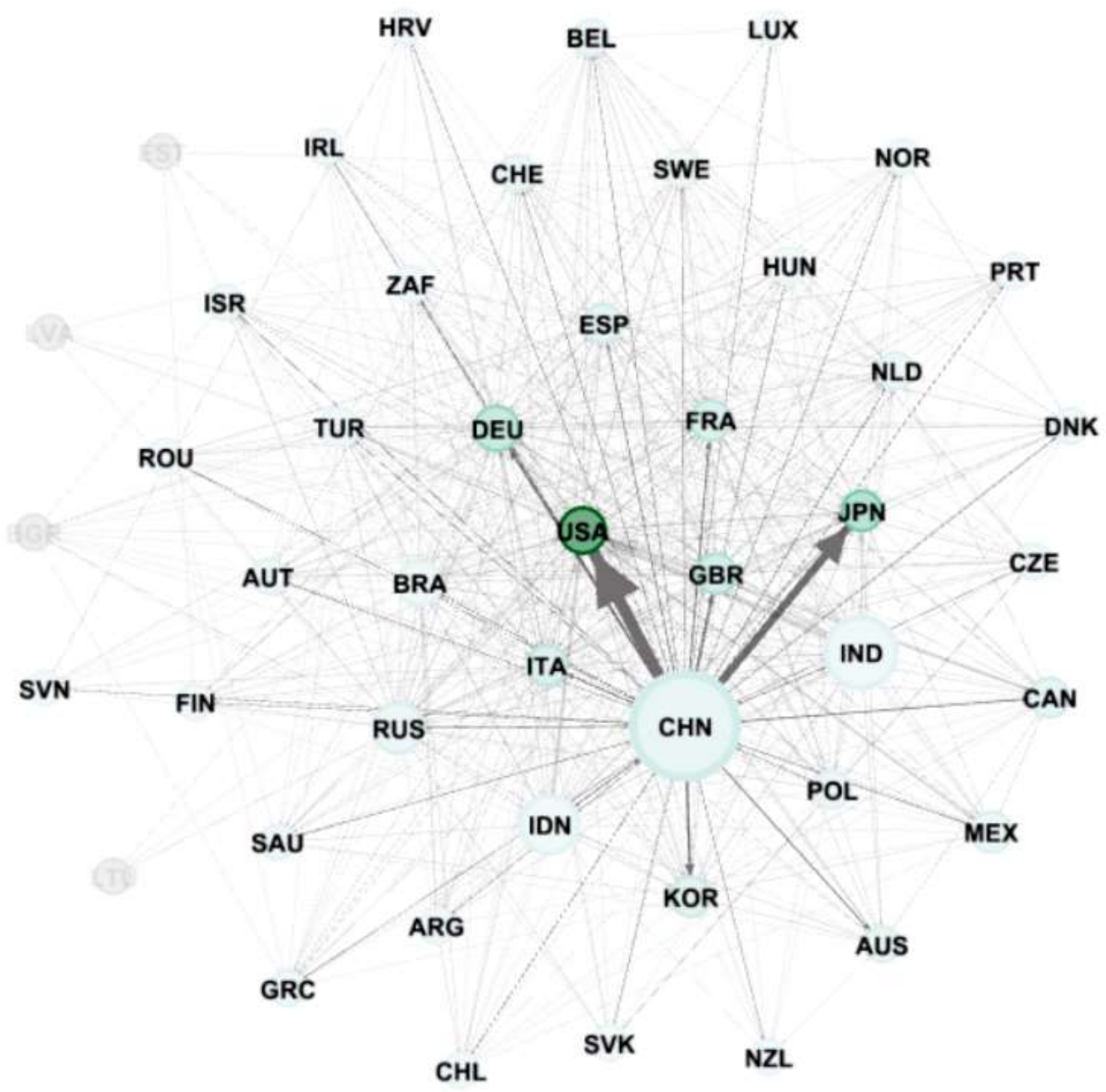

Fuente: OECD/WTO; cálculo del autor 
Figura 7. China: oferta/demanda de empleo para la producción internacional de bienes y servicios finales, 2006

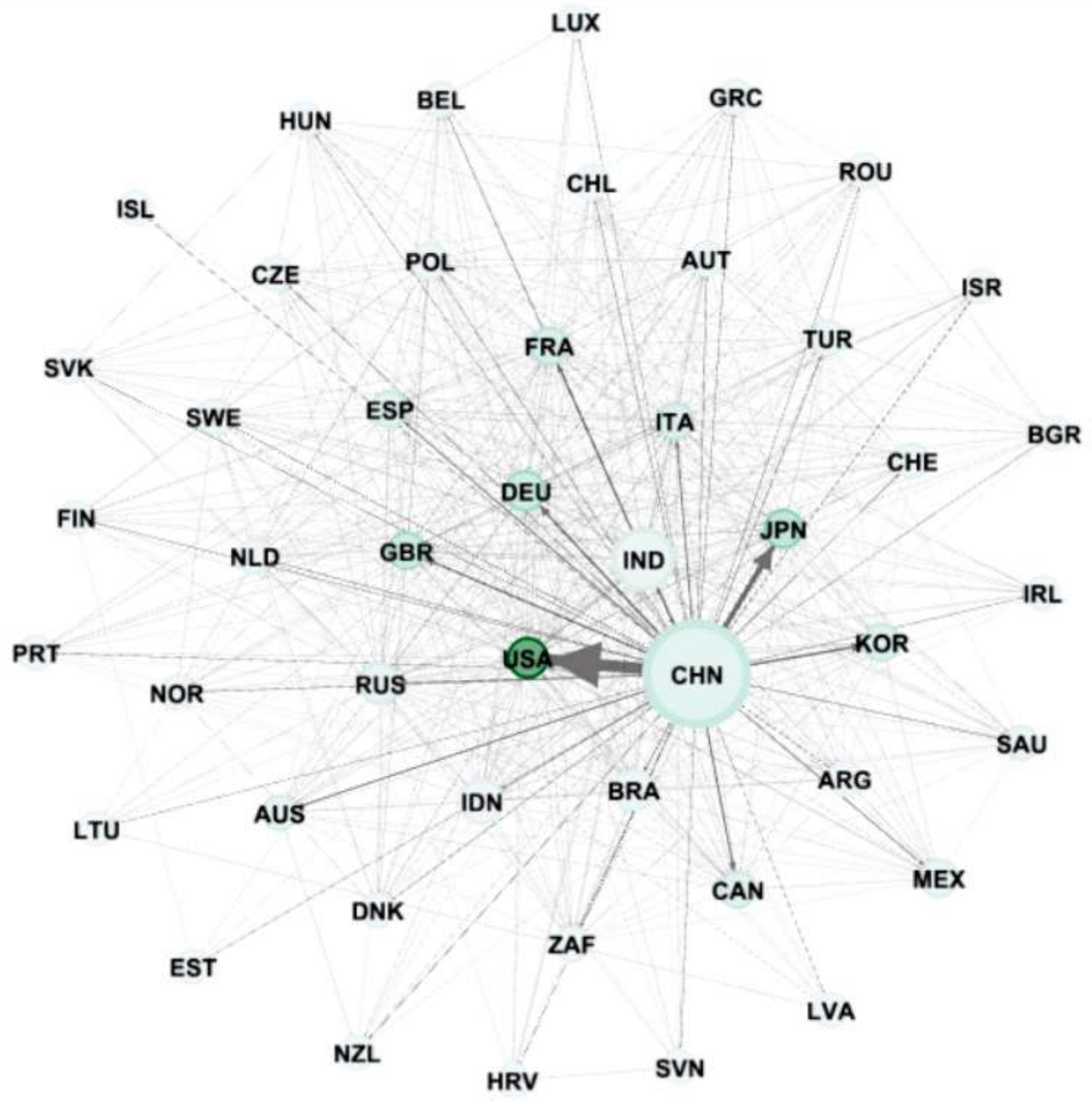

Fuente: OECD/WTO; cálculo del autor 
Figura 8. China: oferta/demanda de empleo para la producción internacional de bienes y servicios finales, 2011

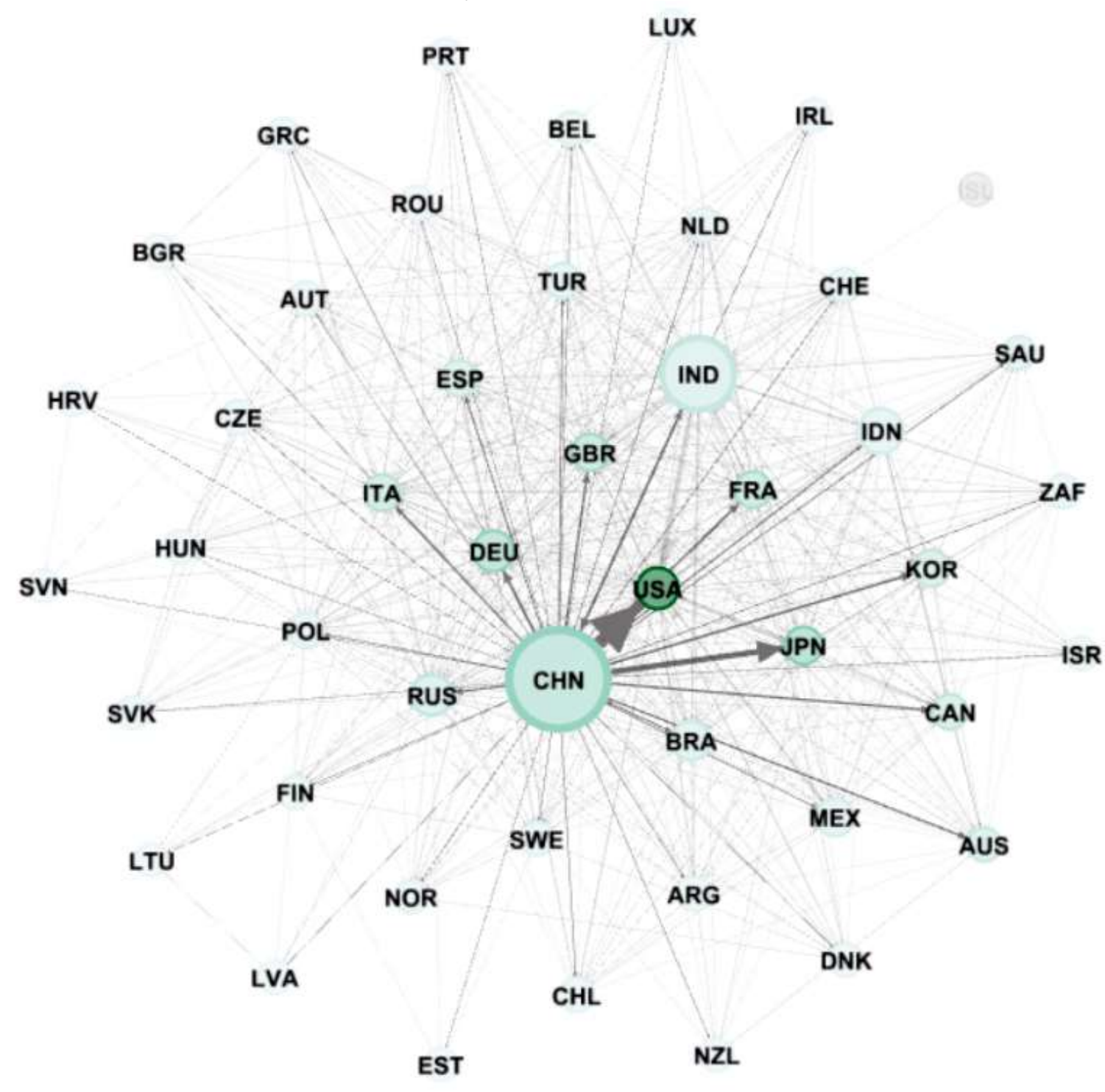

Fuente: OECD/WTO; cálculo del autor 


\section{Conclusiones}

Con base en los datos de Comercio en Empleo (TiM) de la OECD/WTO (2018) de 1995 a 2011, los objetivos de esta investigación fueron: mapear la oferta/demanda de empleo para la producción internacional de bienes y servicios finales y, de esta forma, cuantificar la importancia relativa de México y China. Para llevar a cabo estos objetivos, se analizaron los datos de Comercio en Empleo como un sistema insumo-producto y, posteriormente, como una red compleja a través de un grafo ponderado dirigido. Con base en lo anterior se propusieron algunos indicadores para cuantificar la importancia relativa de México y China en la oferta/demanda de empleo para la producción internacional de bienes y servicios finales.

Los resultados señalan que, en promedio, el 8.58\% del empleo necesario para satisfacer la producción de bienes y servicios finales de México es de origen externo y $91.42 \%$, de origen interno; mientras que China necesita $1.13 \%$ de empleo externo y $98.87 \%$, de empleo interno. Por otro lado, en promedio, el 14.33\% del empleo en México es para satisfacer la demanda final externa y el $85.67 \%$, para la demanda final interna; mientras que el $10.52 \%$ del empleo en China es para la demanda final externa y $89.48 \%$, para la demanda final interna.

Finalmente, los indicadores de posición sugieren que, de forma sistemática, los niveles de empleo de China y México son dependientes de la demanda internacional de bienes y servicios finales, pero independientes del empleo externo para satisfacer su propia demanda final; sin embargo, comparado con otros países, el resto del mundo requiere más empleo chino para satisfacer su demanda final total; además, a diferencia de otros países, la proporción de personas empleadas en la producción de bienes y servicios finales para Estados Unidos en China es la más alta.

De forma congruente, los mapas de oferta/demanda de empleo para la producción internacional de bienes y servicios finales sugieren que la relación entre el comercio internacional y el empleo no sólo es estrecha, sino también compleja: importa la posición, así como los vínculos y socios de cada país. Como lo advierte el Neoestructuralismo, aunque en la producción internacional de bienes y servicios finales existen países centrales que demandan el empleo de países periféricos, los resultados económicos de todas las relaciones de intercambio son, en general, heterogéneos. Comparado con México, la participación de China en la red es mayor: en promedio, la China fue $8.55 \%$, mientras que la de 
México, 3.66\%; es decir, la subred de China es más densa que la de México. Lo anterior evidencia las distintas capacidades de respuesta ante los estímulos externos. Si parafraseamos a George Orwell: todos los países periféricos son iguales, pero algunos son más iguales que otros.

Sin embargo, no sólo es asunto de espacio, sino también de tiempo: la creación de nuevas y mejores relaciones comerciales entre los países puede tomar años. Es necesario detectar los sectores con ventajas comparativas para mejorar el acceso a mercados externos, lo cual exige la sinergia entre política industrial y política de exportación. Además, como señalan Dussel y Armony (2018) y Jansen y Lee (2007), otros factores sincrónicos influyen en estos vínculos dinámicos: cambios tecnológicos, productividad, instituciones, políticas comerciales, etc. Algunos temas pendientes para futuras investigaciones: comercio internacional y calidad del empleo, y comercio internacional, empleo y desigualdad. 


\section{Referencias bibliográficas}

Albert, R. and A.-L. Barabási (2002), "Statistical mechanics of complex networks", Reviews of Modern Physics, 74(1): 47-97. DOI: 10.1103/RevModPhys.74.47

Barrat, A., Barthélémy, M., Pastor-Satorras, R., and Vespignani, A. (2004), "The architecture of complex weighted networks", Proceedings of the National Academy of Sciences, 101 (11), 3747-3752. DOI: 10.1073/pnas.0400087101

Bielschowsky, R. (2013), Neo-estructuralismo vs. estructuralismo clásico: Semejanzas, avances e insuficiencias, en: Seminario sobre Neoestructuralismo y Economía Heterodoxa organizado por CEPAL, 22 y 23 de abril de 2013, Santiago de Chile, 2013.

Cerina, F., Zhu, Z., Chessa, A., and Riccaboni, M. (2015), "World InputOutput Network", PLoS ONE, 10(7): e0134025. doi: 10.1371/journal.pone.0134025

Christensen, C. and Albert, R. (2007), "Using graph concepts to understand the organization of complex systems", Int. J. Bifurcation Chaos, 17, 2201. DOI: http://dx.doi.org/10.1142/S021812740701835X

Dussel, E. y A. Armony (2018), Efectos de China en la cantidad y calidad del empleo generado en América Latina (2000-2017), Organización Internacional del Trabajo.

Gallagher, K., and Porzecanski, R. (2008), "China matters: China's economic impact in Latin America", Latin American Research Review, Vol. 43, No. 1:185-200.

García-Ramos, M. (2018), "Generación de valor agregado en la Red Mundial de Comercio: las ganancias de México y China, 1995-2011”, Centro de Estudios China-México, mimeo.

García-Ramos, M. y Fujii-Gambero, G. (2017), "Origen y destino del Valor Agregado contenido en las exportaciones de Argentina, Brasil, Colombia, Costa Rica, Chile y México, en Banco Central de Bolivia”, Balance del pensamiento económico latinoamericano, Bolivia. 
Gereffi, G. and Fernandez-Stark, K. (2011), Global Value Chain Analysis: A Primer. Center on Globalization, Governance \& Competitiveness (CGGC), Duke University, North Carolina, USA. Available at: http://www.cggc.duke.edu/pdfs/2011-05-31_GVN_analysis_a_primer.pdf

Gibson, B. (2011), "Assessing the impact of trade on employment: Methods of análisis", en Marion Jensen, Ralf Peters y José Manuel Salazar-Xirinachs (directores): Trade and Employment: From Myths to Facts. Ginebra, OIT.

Jacomy, M. T. Venturini, S. Heymann, and M. Bastian (2014): "ForceAtlas2: a Continous Graph Layout Algorithm for Handy Network Visualization Designed for the Gephi Software", PLoS ONE, 9(6).

Jansen, M. y E. Lee (2007), Comercio y empleo. Los retos de la investigación sobre las políticas, Organización Internacional del Trabajo y Organización Mundial del Comercio.

Jansen, M., R. Peters, and J.M. Salazar-Xirinachs (2011), Trade and Empleoyment. From Myths to Facts, OIT, Ginebra.

Jiang, X. (2015), "Efectos en el empleo del comercio de bienes intermedios y finales. Una evaluación empírica", Revista Internacional del Trabajo, vol. 134 (2015), núm. 2

Lall, S. and Weiss, J. (2007), "China and Latin America: trade competition, 1990-2002", in Santiso, J. (ed), The visible hand of China in Latin America, OECD Publishing, Paris, pp 85-10.

Maurer, A. and Degain, C. (2010), "Globalization and trade flows: what you see is not what you get!", Staff Working Paper, ERSD-2010-12. Available at: http://dx.doi.org/10.1142/S1793993312500196

McMillan, M. and Verduzco, I. (2011), "New Evidence on Trade and Employment: An Overview", en Marion Jensen, Ralf Peters y José Manuel Salazar-Xirinachs (directores): Trade and Employment: From Myths to Facts. Ginebra, OIT.

OECD/WTO (2018), "Trade in employment", OECD-WTO: Statistics on Trade in Employment (database). 
Prebisch, R. (1949), El desarrollo económico de la América Latina y algunos de sus principales problemas, (E/CN.12/89), Santiago de Chile, Comisión Económica para América Latina (CEPAL).

Schwarzer, J. (2016), "Trade and Employment. An Overview," Discussion Notes 1601, Council on Economic Policies.

Sunkel, O. y P. Paz (1970), El subdesarrollo latinoamericano y la teoría del desarrollo, Siglo XXI, México.

Taglioni, D. and Winker, D. (2016), Making Global Value Chains Work for Development, Trade and Development series, Washington, DC: World Bank. DOI:10.1596/978-1-4648-0157-0

UNCTAD (2013), "Efectos del comercio en la creación de empleo y la reducción de la pobreza", Conferencia de las Naciones Unidas sobre Comercio y Desarrollo. 


\section{Anexo}

Tabla 1A. Nomenclatura de países

\begin{tabular}{|c|c|c|c|}
\hline Id & País & Id & País \\
\hline $\mathrm{ARG}$ & Argentina & ITA & Italia \\
\hline AUS & Australia & JPN & Japón \\
\hline AUT & Austria & KHM & Camboya \\
\hline BEL & Bélgica & KOR & Corea \\
\hline BGR & Bulgaria & LTU & Lituania \\
\hline BRA & Brasil & LUX & Luxemburgo \\
\hline $\mathrm{BRN}$ & Brunéi Darussalam & LVA & Letonia \\
\hline $\mathrm{CAN}$ & Canadá & MEX & México \\
\hline $\mathrm{CHE}$ & Suiza & MLT & Malta \\
\hline $\mathrm{CHL}$ & Chile & MYS & Malasia \\
\hline $\mathrm{CHN}$ & China & NLD & Países Bajos \\
\hline $\mathrm{COL}$ & Colombia & NOR & Noruega \\
\hline CRI & Costa Rica & NZL & Nueva Zelanda \\
\hline CYP & Chipre & PER & Perú \\
\hline $\mathrm{CZE}$ & República Checa & PHL & Filipinas \\
\hline DEU & Alemania & POL & Polonia \\
\hline DNK & Dinamarca & PRT & Portugal \\
\hline ESP & España & ROU & Rumania \\
\hline $\mathrm{EST}$ & Estonia & RUS & Rusia \\
\hline FIN & Finlandia & SAU & Arabia Saudita \\
\hline FRA & Francia & SGP & Singapur \\
\hline GBR & Reino Unido & SVK & República Eslovaca \\
\hline GRC & Grecia & SVN & Eslovenia \\
\hline $\mathrm{HKG}$ & Hong Kong & SWE & Suecia \\
\hline $\mathrm{HRV}$ & Croacia & THA & Tailandia \\
\hline HUN & Hungría & TUN & Túnez \\
\hline IDN & Indonesia & TUR & Turquía \\
\hline IND & India & TWN & Taiwán \\
\hline IRL & Irlanda & USA & Estados Unidos \\
\hline ISL & Islandia & VNM & Vietnam \\
\hline ISR & Israel & $\mathrm{ZAF}$ & Sudáfrica \\
\hline
\end{tabular}

